\title{
Pressure drop coefficient of laminar Newtonian flow in axisymmetric diffusers
}

\author{
S. Rosa ${ }^{a}$, F.T. Pinho ${ }^{b, *}$ \\ ${ }^{\text {a }}$ Escola Superior de Tecnologia e Gestão, Instituto Politécnico, Campus de Santa Apolónia, 5301-857 Bragança, Portugal \\ ${ }^{\mathrm{b}}$ Centro de Estudos de Fenómenos de Transporte, DEM, Universidade do Minho, Campus de Azurém, 4800-058 Guimarães, Portugal
}

\begin{abstract}
The laminar flow of Newtonian fluids in axisymmetric diffusers has been numerically investigated to evaluate the pressure-loss coefficient as a function of Reynolds number, diffusion angle and expansion ratio. The numerical simulations were carried out with a finitevolume based code using non-orthogonal collocated grids and second order accurate differencing schemes to discretize all terms of the transport equations.

The calculations were carried out for Reynolds numbers between 2 and 200, diffusion angles from $0^{\circ}$ to $90^{\circ}$ and expansion ratios of 1.5 and 2 and the data are presented in tabular form and as correlations. A simplified 1D theoretical analysis helped explain the various contributions to the loss coefficient and its difference relative to the reversible pressure variation due to differences between the actual and fully developed friction losses, distortions of the velocity profiles and pressure non-uniformity upstream and downstream of the expansion section.
\end{abstract}

Keywords: Irreversible loss coefficient; Axisymmetric diffusers; Laminar flow; Numerical

\section{Introduction}

Many industrial applications require piping systems to provide energy and deliver products and often these take place under laminar flow conditions for sub-critical Reynolds numbers. It is also generally the case for miniaturized fluid mechanical devices where the operation is almost exclusively under laminar flow conditions. A common component of such systems is the expansion, which can be sudden or gradual, and it is necessary to understand in more detail its laminar flow characteristics, especially for the diffuser for which the literature is scarce. In engineering calculations it is usually the pressure drop, the pipe velocity, and/or the pipe diameter that has to be deter- mined and this requires accurate values for the loss coefficient.

Classical fluid dynamics textbooks and references such as Runstadler et al. (1975), Crane Co. (1979), Gibson (1930), Massey (1989) and Tsui and Wang (1995), all present the same expressions for the local loss coefficient in diffusers which were derived on the basis of an inlet uniform velocity profile and negligible shear stresses. That expression compares well with data for turbulent flow, but is not appropriate for laminar flow due to non-negligible shear stresses, amongst other things.

This study is a numerical investigation on laminar diffuser flows of Newtonian fluids aimed at quantifying the irreversible loss coefficient $C_{\mathrm{I}}$ and extends previous research of Oliveira and Pinho (1997) on sudden expansion flows at low Reynolds numbers. For sudden expansions they found large discrepancies between their local loss coefficient and the expressions from the literature, which were also based on fast flow redevelopment, negligible shear 


\section{Nomenclature}

A cross-section area

$C \quad$ normalised pressure coefficient, $C \equiv \frac{p}{\frac{1}{2} \rho \bar{u}_{1}^{2}}$

$\Delta C_{\mathrm{F}}$ friction contribution into the irreversible loss coefficient

$\Delta C_{\mathrm{I}} \quad$ correction to theoretical loss coefficient

$\Delta C_{\beta} \quad$ distortions of velocity profiles

$\Delta C_{p 0} \quad$ nonuniform pressure effects

$C_{\mathrm{F}} \quad$ fully developed pressure coefficient

$C_{\mathrm{I}} \quad$ irreversible pressure coefficient

$C_{\mathrm{Ic}} \quad$ corrected irreversible pressure coefficient

$C_{\text {Ith }}$ theoretical irreversible pressure coefficient

$C_{p} \quad$ pressure coefficient

$\frac{C_{p 01}}{C_{p 02}}, \overline{C_{p 02}}$ normalised area-averaged pressure coefficient at cross-sections 01 and 02 , respectively

$C_{\mathrm{R}} \quad$ reversible pressure coefficient

$C_{\mathrm{T}} \quad$ total pressure coefficient

$D_{1}, D_{2}$ diameter of inlet and outlet pipes, respectively

$f \quad$ Darcy friction factor

$f_{x}, f_{y}, f_{z}$ geometrical expansion factors for mesh spacing

$L \quad$ length
$N_{x}, N_{y}, N_{z}$ number of internal cells of computational grid

$p_{2}, p_{1} \quad$ pressure at inlet and outlet planes respectively

$\Delta p_{\mathrm{F}} \quad$ fully developed pressure drop

$\Delta p_{\mathrm{F}}^{\prime} \quad$ actual wall friction pressure drops

$\Delta p_{\mathrm{I}} \quad$ irreversible pressure drop

$\Delta p_{\mathrm{R}} \quad$ reversible pressure decrease

$\Delta p_{\mathrm{T}} \quad$ total pressure drop

$u \quad$ axial velocity component

$\overline{u_{1}}, \overline{u_{2}}$ bulk velocity in the inlet and outlet pipes

$X_{1 a}, X_{1 b}$ mark beginning and end of fully-developed flow in the upstream pipe

$X_{2 S} \quad$ marks beginning of region of fully-developed flow in downstream pipe

$\alpha, \beta \quad$ profile shape factors for energy $\left(\alpha \equiv \overline{u^{3}} / \bar{u}^{3}\right)$ and momentum $\left(\beta \equiv \overline{u^{2}} / \bar{u}^{2}\right)$

$\theta \quad$ half-angle of diffuser

$\mu \quad$ fluid dynamic viscosity

$\rho \quad$ fluid density

$\sigma \quad$ area ratio $\left(\equiv D_{1}^{2} / D_{2}^{2}\right)$ stresses and uniform velocities at inlet and outlet. The suspicion that a similar situation would occur for diffusers motivated the present work.

More specifically, the purpose here is to numerically evaluate the variation of $C_{\mathrm{I}}$ in diffusers as a function of the Reynolds number, diffuser angle $(\alpha)$ and expansion ratio $\left(D_{2} / D_{1}\right)$, using a finite volume code. We will also attempt to explain and understand the variations found, using one-dimensional energy and momentum balances.

The next section presents this one-dimensional theory and defines the problem and relevant quantities. This is followed by an outline of the numerical procedure and the specification of the calculation domain and boundary conditions. The presentation and discussion of the numerical results are preceded by an assessment of their uncertainties and by validation and verification against other computed quantities, experimental data and correlations from the literature. A useful correlation for the local loss coefficient is proposed at the end before the summary of the main conclusions.

\section{One-dimensional theory}

We concentrate on laminar flow in an axisymmetric gradual expansion with fully-developed conditions at the inlet pipe, which is located far upstream of the diffuser in order for the flow to adapt more realistically to the geometry. A schematic representation of the control volumes used in the following one-dimensional theory is shown in Fig. 1. This theoretical analysis is an adaptation of that derived by Oliveira and Pinho (1997) for sudden expansions.

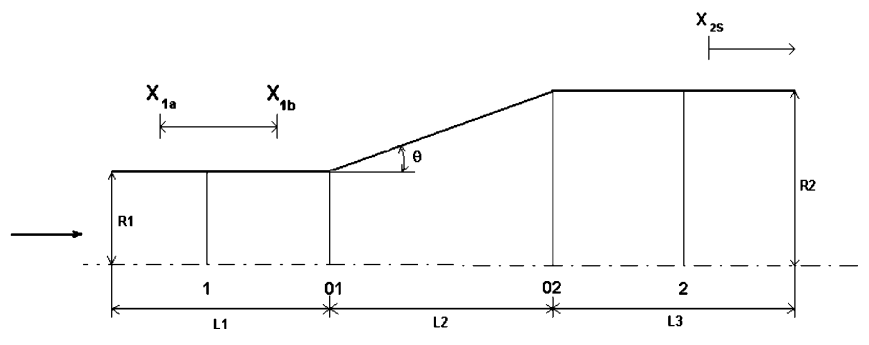

Fig. 1. Gradual expansion geometry and its control volume.

In pressure drop calculations, it is engineering practice to consider that the flow is fully developed in straight pipes or ducts, with all other effects, such as flow distortions and flow redevelopment downstream of fittings introduced via their respective local loss coefficients. The total pressure variation between cross-section planes 1 and 2 (see Fig. 1) is decomposed into a reversible pressure increase $\left(\Delta p_{\mathrm{R}}\right)$, an irreversible pressure drop $\left(\Delta p_{\mathrm{I}}\right)$, and the pressure variation due to fully developed friction on the upstream and downstream pipes $\left(\Delta p_{\mathrm{F}}\right)$. After normalization with the upstream dynamic pressure $\left(1 / 2 \rho \bar{u}_{1}^{2}\right)$, this decomposition is written as

$C_{\mathrm{T}}=C_{\mathrm{R}}-C_{\mathrm{I}}-C_{\mathrm{F}}=C_{\mathrm{RI}}-C_{\mathrm{I}}$

Note that $C_{\mathrm{I}}$ includes a friction effect, because the actual friction between planes 1 and $2\left(\Delta p_{\mathrm{F}}^{\prime}\right)$ is different from the corresponding fully developed friction $\left(\Delta p_{\mathrm{F}}\right)$.

Integral conservation of longitudinal momentum applied to the control volumes between stations 1 and 01 , and planes 02 and 2 of Fig. 1 are expressed by Eqs. (2) and (3), respectively. 
$p_{1} A_{1}+\rho A_{1} \beta_{1}{\overline{u_{1}}}^{2}=\overline{p_{01}} A_{1}+\rho A_{1} \beta_{01} \overline{\overline{01}}^{2}+\int \tau_{01-1} \cdot \mathrm{d} S_{1}$

$\overline{p_{02}} A_{2}+\rho A_{2} \beta_{02}{\overline{u_{02}}}^{2}=p_{2} A_{2}+\rho A_{2} \beta_{02}{\overline{u_{2}}}^{2}+\int \tau_{02-2} \cdot \mathrm{d} S_{2}$

where the profile shape factor for momentum, $\beta \equiv \overline{u^{2}} / \bar{u}^{2}$ is used. $\tau_{01-1}$ and $\tau_{02-2}$ represent the local wall shear stress between planes 01 and 1 and planes 02 and 2, respectively, and the overbar denotes area-averaged quantities. The integrals of the wall shear stresses are transformed into pressure differences as in Eq. (4)

$$
\begin{aligned}
& \int \tau_{01-1} \cdot \mathrm{d} S_{1}=\overline{\tau_{01-1}} \cdot S_{1}=\Delta p_{\mathrm{F} 1}^{\prime} \cdot A_{1} \quad \text { and } \\
& \int \tau_{02-2} \cdot \mathrm{d} S_{2}=\overline{\tau_{02-2}} \cdot S_{2}=\Delta P_{\mathrm{F} 2}^{\prime} \cdot A_{2}
\end{aligned}
$$

with $S_{1}$ and $S_{2}$ representing the pipe wall area acted by area-averaged shear stresses $\overline{\tau_{01-1}}$ and $\overline{\tau_{02-2}}$, respectively.

Defining the area ratio, $\sigma \equiv A_{1} / A_{2}$, and considering mass conservation $\left(A_{1} \overline{u_{1}}=A_{2} \overline{u_{2}}\right)$, the combination of the above momentum balances as (Eq. (2)) $+\sigma$ (Eq. (3)) leads to Eq. (5) after division by $A_{1}$ and the upstream kinetic energy

$$
\begin{aligned}
C_{\mathrm{T}} \equiv & \frac{p_{2}-p_{1}}{\frac{1}{2} \cdot \rho \cdot{\overline{u_{1}}}^{2}}=2\left(\beta_{1}-\beta_{01}\right)-2 \sigma^{2} \cdot\left(\beta_{2}-\beta_{02}\right) \\
& -\frac{\Delta p_{\mathrm{F} 2}^{\prime}+\Delta p_{\mathrm{F} 1}^{\prime}+\left(\overline{p_{01}}-\overline{p_{02}}\right)}{\frac{1}{2} \cdot \rho \cdot{\overline{u_{1}}}^{2}}
\end{aligned}
$$

The total pressure coefficient is given in Eq. (1) and the reversible pressure coefficient $\left(C_{\mathrm{R}}=\alpha_{1}-\alpha_{2} \sigma^{2}\right)$ is obtained from the energy equation assuming a reversible flow (Bernoulli equation), where the profile shape factor for energy is $\alpha \equiv \overrightarrow{u^{3}} / \bar{u}^{3}$. Combining $C_{\mathrm{R}}$ with Eqs. (1) and (5) gives the corrected loss coefficient based on this approximate 1-D theory $\left(C_{\mathrm{Ic}}\right)$, which is different from the correct loss coefficient $\left(C_{\mathrm{I}}\right)$ obtained in numerical simulations with the full set of two-dimensional momentum and continuity equations

$$
\begin{aligned}
C_{\mathrm{Ic}}= & \alpha_{1}-\alpha_{2} \sigma^{2}-C_{\mathrm{F}}-2\left(\beta_{1}-\beta_{01}\right)+2 \sigma^{2} \cdot\left(\beta_{2}-\beta_{02}\right) \\
& +C_{\mathrm{F} 2}^{\prime}+C_{\mathrm{F} 1}^{\prime}+\overline{C_{p 01}}-\overline{C_{p 02}}
\end{aligned}
$$

This expression can be cast in the form of a sum of corrections to the reversible pressure coefficient

$C_{\mathrm{Ic}}=C_{\mathrm{R}}-\left(\Delta C_{\mathrm{F}}+\Delta C_{\beta}-\Delta C_{p 0}\right)$

where $\Delta C_{\mathrm{F}}$ represents the difference between the fully developed friction $C_{\mathrm{F}}$ and the actual friction $C_{\mathrm{F}^{\prime}}$ due to a variable wall shear stress in the downstream and upstream pipes, respectively, i.e.

$\Delta C_{\mathrm{F}}=\Delta C_{\mathrm{F} 1}+\Delta C_{\mathrm{F} 2} \quad$ with

$\Delta C_{\mathrm{F} 2}=C_{\mathrm{F} 2}-C_{\mathrm{F} 2}^{\prime}$ and $\Delta C_{\mathrm{F} 1}=C_{\mathrm{F} 1}-C_{\mathrm{F} 1}^{\prime}$.
The fully-developed and the actual friction coefficients are given by

$$
\begin{aligned}
& C_{\mathrm{F} 1}=\frac{\Delta p_{\mathrm{F} 1}}{\frac{1}{2} \rho{\overline{u_{1}}}^{2}}=\frac{f_{1} \frac{L_{1}}{\bar{D}_{1}} \frac{{\overline{u_{1}}}^{2}}{2} \rho}{\frac{1}{2} \rho{\overline{u_{1}}}^{2}}=f_{1} \frac{L_{1}}{D_{1}}, \quad C_{\mathrm{F} 2}=\frac{\Delta p_{\mathrm{F} 2}}{\frac{1}{2} \rho{\overline{u_{1}}}^{2}}=\sigma^{2} f_{2} \frac{L_{3}}{D_{2}} \\
& C_{\mathrm{F} 1}^{\prime}=\frac{\Delta p_{\mathrm{F} 1}^{\prime}}{\frac{1}{2} \rho{\overline{u_{1}}}^{2}}=\frac{4 L_{1}}{D_{1}} \frac{\overline{\tau_{W 1}}}{\frac{1}{2} \rho{\overline{u_{1}}}^{2}}, \quad C_{\mathrm{F} 2}^{\prime}=\frac{\Delta p_{\mathrm{F} 2}^{\prime}}{\frac{1}{2} \rho{\overline{u_{1}}}^{2}}=\frac{4 L_{3}}{D_{2}} \frac{\overline{\tau_{W 2}}}{\frac{1}{2} \rho{\overline{u_{1}}}^{2}}
\end{aligned}
$$

$\Delta C_{\beta}$ accounts for the differences in momentum immediately upstream and downstream of the gradual expansion $\left(\Delta C_{\beta}=\Delta C_{\beta 1}+\Delta C_{\beta 2}\right)$ due to distortions of the velocity profiles. For a parabolic velocity profile $\alpha_{1}=\alpha_{2}=2$, and $\beta_{1}=\beta_{2}=4 / 3$ leading to:

$\Delta C_{\beta 1}=2\left(\beta_{1}-\beta_{01}\right)=2\left(\frac{4}{3}-\beta_{01}\right) \quad$ and

$\Delta C_{\beta 2}=-2 \sigma^{2}\left(\beta_{2}-\beta_{02}\right)=-2 \sigma^{2}\left(\frac{4}{3}-\beta_{02}\right)$

Finally, $\Delta C_{p 0}$ quantifies the effect of non-uniform pressure at the planes immediately upstream and downstream of the expansion

$\Delta C_{p 0}=\left(\overline{C_{p 01}}-\overline{C_{p 02}}\right)$

To determine these corrections from the results of the numerical simulations, $\beta_{0 i}$ and $\overline{C_{p 0 i}}$ are calculated by numerical integration of the velocity and pressure profiles at the plane $0 i(i=1,2)$, respectively. Note that Eq. (7) is a simplified method to quantify the local loss coefficient but still it can be used to help understand its various contributions.

The correct irreversible coefficient $\left(C_{\mathrm{I}}\right)$ is determined from the axial variation of pressure obtained in the numerical solution of the full Navier-Stokes equations, as follows: the energy equation between stations 1 and 2 (see Fig. 1) reads as

$$
\begin{aligned}
P_{1}+\frac{1}{2} \rho \alpha_{1} V_{1}^{2}+\rho g Z_{1}= & p_{2}+\frac{1}{2} \rho \alpha_{2} V_{2}^{2}+\rho g Z_{2}+\frac{1}{2} \rho V_{1}^{2} \cdot C_{\mathrm{I}} \\
& +f_{1} \frac{L_{1}}{D_{1}} \rho \frac{V_{1}^{2}}{2}+f_{2} \frac{L_{3}}{D_{2}} \rho \frac{V_{2}^{2}}{2}
\end{aligned}
$$

After simplification we obtain the following extrapolated pressures at planes 01 and 02 , respectively by fitting to pressure variations only along the fully-developed regions upstream and downstream of the diffuser: $\overline{\overline{p_{01}}} \equiv$ $p_{1}-f_{1} \frac{L_{1}}{D_{1}} \rho \frac{V_{1}^{2}}{2}$ and $\overline{\overline{p_{02}}} \equiv p_{2}+f_{2} \frac{L_{3}}{D_{2}} \rho \frac{V_{2}^{2}}{2}$. Then, we calculate $C_{\mathrm{I}}$ using Eq. (13)

$C_{\mathrm{I}}=\frac{\overline{\overline{p_{01}}}-\overline{\overline{p_{02}}}}{\frac{1}{2} \rho{\overline{u_{1}}}^{2}}+1-\sigma^{2}=\left(\overline{\overline{C_{p 01}}}-\overline{\overline{C_{p 02}}}\right)+1-\sigma^{2}$

This method is identical to that used by Oliveira and Pinho (1997) in the context of sudden expansions.

The loss coefficient usually found in books $\left(C_{\mathrm{Ith}}\right.$ in Eq. (14)) was derived assuming inlet and outlet uniform velocity profiles and negligible shear stresses within the domain. The multiplicative factor $2.6 \sin \theta$ was obtained by Gibson 
(1930) for small diffuser angles and is equal to 1 in the other cases. This coefficient is a good approximation for turbulent flows according to Shames (1992), but leads to values widely in error for laminar flow, as will be shown.

$$
C_{\text {I th }}= \begin{cases}(1-\sigma)^{2} 2.6 \sin \theta & \theta \leqslant 22.5^{\circ} \\ (1-\sigma)^{2} & 22.5^{\circ}<\theta \leqslant 90^{\circ}\end{cases}
$$

The difference between this coefficient and the corrected pressure drop, Eq. (7) can be better understood by rewriting $C_{\text {Ic }}$ as

$C_{\text {Ic }}=C_{\text {Ith }}-\Delta C_{\text {I }}$

with $\Delta C_{\mathrm{I}}=\Delta C_{\mathrm{F}}+\Delta C_{\beta}-\Delta C_{p 0}+\Delta C_{\theta}$ and $\Delta C_{\theta}$ given by

$$
\Delta C_{\theta}= \begin{cases}(1-\sigma)^{2} 2.6 \sin \theta-C_{\mathrm{R}} & \\ =(1-\sigma)^{2} 2.6 \sin \theta-2\left(1-\sigma^{2}\right) & \theta \leqslant 22.5^{\circ} \\ (1-\sigma)^{2}-C_{\mathrm{R}}=(1-\sigma)^{2}-2\left(1-\sigma^{2}\right) & 22.5^{\circ}<\theta \leqslant 90^{\circ}\end{cases}
$$

\section{Numerical procedure, uncertainties and validation}

The numerical calculations were carried out with a standard finite-volume code extensively described by Issa and Oliveira (1992) and briefly explained here. The mass and momentum differential transport equations were discretised by a control volume based finite difference scheme described in Patankar (1980), and later adapted by Peric (1985) for non-staggered, non-orthogonal grids. The main code is interfaced with a mesh generation pre-processor as described by Oliveira (1992) and adequate data postprocessors. The basic differencing schemes were all second order accurate: central differencing for the diffusion terms and the Linear Upwind Differencing Scheme (LUDS), also called Second Order Upwind, for the convective terms. For stability reasons, the convective flux was calculated explicitly from values of the previous iteration and combined with first order convective fluxes using the first order Upwind Differencing Scheme following the deferred correction technique of Khosla and Rubin (1974). The pressure-velocity coupling was based on the SIMPLEC algorithm of Van Doormal and Raithby (1984) and modified by Issa and Oliveira (1992) to account for time-marching, since this steady flow calculation was stabilised by a pseudo-transient method instead of under-relaxation. The discretized equations were solved iteratively using the conjugate gradient method preconditioned with an incomplete LU decomposition for the pressure and the bi-conjugate gradient method for the velocities.

Tests with different meshes were initially performed to assess the adequacy of the computational domain and grid to obtain accurate and grid independent results. For simplicity a uniform velocity was set at the inlet and the flow allowed to develop well upstream the beginning of the diffuser. The grids, shown in Fig. 2, were built from three patched structured blocks and two types of grids were used for diffuser angles $(\theta)$ less and larger than $45^{\circ}$, respectively. In both cases the first block corresponded to the inlet pipe. For $\theta<45^{\circ}$ (see Fig. 2(a)), the second block corresponded to the expansion zone and the third block mapped the outlet pipe, whereas for $\theta>45^{\circ}$ (see Fig. 2(b)) the second block only mapped the geometry downstream of the first block, and the third block mapped the region downstream the expansion wall.

The inlet pipe diameter was $10 \mathrm{~mm}$ and its length was 50 diameters $\left(L_{1}=50 D_{1}\right)$. The length of the outlet pipe was $L_{3}=100 D_{1}$ and two diameter ratios of $1: 1.5$ and $1: 2$ were investigated. The diffuser length $L_{2}$ changed with the expansion ratio and diffuser angle, but the number of computational cells within the diffuser was such as to maintain the required mesh fineness. The calculations were carried out for Reynolds numbers ranging from 2 to 200, as it is known that for $R e>200$ the first instabilities appear in the flow downstream of the expansion. The Reynolds
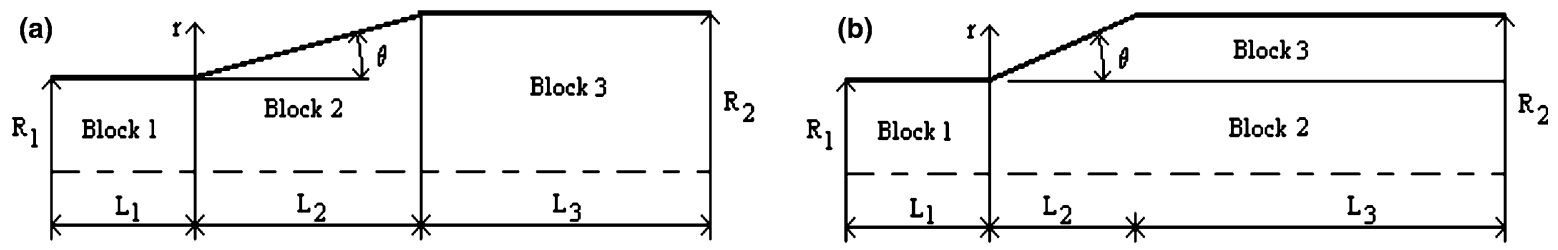

Fig. 2. Computational domain for: (a) $\alpha<45^{\circ}$; (b) $\beta>45^{\circ}$.

Table 1

\begin{tabular}{|c|c|c|c|c|c|c|}
\hline \multirow[t]{2}{*}{ Grid } & \multicolumn{2}{|l|}{ Block I } & \multicolumn{2}{|l|}{ Block II } & \multicolumn{2}{|l|}{ Block III } \\
\hline & $N_{x} / f_{x}$ & $N_{y} / f_{y}$ & $N_{x} / f_{x}$ & $N_{y} / f_{y}$ & $N_{x} / f_{x}$ & $N_{y} / f_{y}$ \\
\hline Coarse & $25 / 0.793293$ & $25 / 1$ & $15 / 1.050443$ & $25 / 1$ & $50 / 1.101989$ & $25 / 1$ \\
\hline Normal & $50 / 0.890670$ & $50 / 1$ & $30 / 1.024911$ & $50 / 1$ & $100 / 1.049757$ & $50 / 1$ \\
\hline Fine & $100 / 0.943753$ & $100 / 1$ & $60 / 1.012379$ & $100 / 1$ & $200 / 1.024576$ & $100 / 1$ \\
\hline
\end{tabular}

Some grid characteristics for $\theta<45^{\circ}\left(D_{2} / D_{1}=2 ; \theta=30^{\circ}\right)$ 
Table 2

Some grid characteristics for $\theta>45^{\circ}\left(D_{2} / D_{1}=2 ; \theta=75^{\circ}\right)$

\begin{tabular}{|c|c|c|c|c|c|c|}
\hline \multirow[t]{2}{*}{ Grid } & \multicolumn{2}{|l|}{ Block I } & \multicolumn{2}{|l|}{ Block II } & \multicolumn{2}{|l|}{ Block III } \\
\hline & $N_{x} / f_{x}$ & $N_{y} / f_{y}$ & $N_{x} / f_{x}$ & $N_{y} / f_{y}$ & $N_{x} / f_{x}$ & $N_{y} / f_{y}$ \\
\hline Coarse & $25 / 0.793293$ & $13 / 1$ & $60 / 1.095687$ & $13 / 1$ & $60 / 1.095687$ & $13 / 1$ \\
\hline Normal & $50 / 0.890670$ & $26 / 1$ & $120 / 1.04675$ & $26 / 1$ & $120 / 1.04675$ & $26 / 1$ \\
\hline Fine & $100 / 0.943753$ & $52 / 1$ & $240 / 1.02311$ & $52 / 1$ & $240 / 1.02311$ & $52 / 1$ \\
\hline
\end{tabular}

Table 3

$C_{\mathrm{I}}$ values for different grids compared with Richardson extrapolation (ER)

\begin{tabular}{|c|c|c|c|c|c|c|c|c|c|c|c|c|c|c|}
\hline \multirow[t]{2}{*}{$\overline{R e}$} & \multicolumn{7}{|c|}{$0=30^{\circ}$ and $D_{2} / D_{1}=2$} & \multicolumn{7}{|c|}{$\theta=75^{\circ}$ and $D_{2} / D_{1}=2$} \\
\hline & Coarse & $\varepsilon_{\text {rel }}(\%)$ & Normal & $\varepsilon_{\text {rel }}(\%)$ & Fine & $\varepsilon_{\text {rel }}(\%)$ & ER & Coarse & $\varepsilon_{\text {rel }}(\%)$ & Normal & $\varepsilon_{\text {rel }}(\%)$ & Fine & $\varepsilon_{\text {rel }}(\%)$ & ER \\
\hline 2 & 13.4261 & 26.6 & 11.3304 & 6.9 & 10.7859 & 1.7 & 10.6026 & 8.1410 & 6.0 & 7.8140 & 1.8 & 7.7139 & 0.5 & 7.6789 \\
\hline 12.5 & 2.2976 & 16.0 & 2.0667 & 4.4 & 2.0021 & 1.1 & 1.9799 & 1.5670 & 4.9 & 1.6017 & 7.2 & 1.5264 & 2.2 & 1.4938 \\
\hline 50 & 1.0463 & 3.9 & 1.0396 & 3.2 & 1.0166 & 1.0 & 1.0070 & 1.1350 & 16.3 & 1.0541 & 8.0 & 0.9981 & 2.2 & 0.9763 \\
\hline 100 & 0.9317 & 0.0 & 0.9531 & 2.3 & 0.9385 & 0.7 & 0.9318 & 0.9980 & 6.8 & 0.9930 & 6.3 & 0.9518 & 1.9 & 0.9345 \\
\hline 150 & 0.9095 & -0.8 & 0.9317 & 1.6 & 0.9218 & 0.5 & 0.9172 & 0.9650 & 1.8 & 0.9600 & 1.3 & 0.9515 & 0.4 & 0.9481 \\
\hline
\end{tabular}

number is here defined on the basis of inlet bulk velocity and pipe diameter.

To estimate the uncertainty of the numerically obtained loss coefficients, calculations of $C_{\mathrm{I}}$ were carried out for two diffusers $\left(\theta=30^{\circ}, 75^{\circ}\right)$ having the same expansion ratio. For each geometry $C_{\mathrm{I}}$ was determined using three consecutively refined meshes (coarse, medium and fine) and it was further improved by using Richardson's deferred approach to the limit (Richardson, 1927). Tables 1 and 2 summarize the grid characteristics listing the number of internal cells in the streamwise $\left(N_{X}\right)$ and radial $\left(N_{Y}\right)$ directions and the corresponding geometric expansion (or contraction) factors for mesh spacing $\left(f_{x}, f_{y}\right)$. These factors enabled mesh refinement in regions where large gradients were expected. Given the azimuthal flow symmetry a single cell was used in this direction with symmetry boundary conditions set at the two corresponding cell faces, i.e, the calculations were $2 \mathrm{D}$.

Table 3 presents results of the calculated $C_{\mathrm{I}}$ in the three meshes and the extrapolated value (ER). All errors $\left(\varepsilon_{\text {rel }}\right)$ were calculated in relation to this extrapolated value and for the finer mesh show uncertainties not exceeding 2.2\% at intermediate Reynolds numbers for $\theta=75^{\circ}$, decreasing to less than $1 \%$ at lower and higher Reynolds numbers. For $\theta=30^{\circ}$ the uncertainties are even lower. Therefore, the finer mesh was used in all calculations to maintain similar levels of accuracy. The calculations were carried out with a Pentium III at $1 \mathrm{GHz}$ and each simulation typically took about 1200 min of CPU time.

For validation we investigated two limiting cases for which there are data in the literature: flow in a diffuser tending to a pipe (i.e. $\theta \rightarrow 0$ and $D_{2} / D_{1} \rightarrow 1$ ) and flow in a sudden expansion $\left(\theta=90^{\circ}\right)$. For the former, Fig. 3 shows the evolution of $C_{\mathrm{I}}$ toward the Darcy friction factor expres-

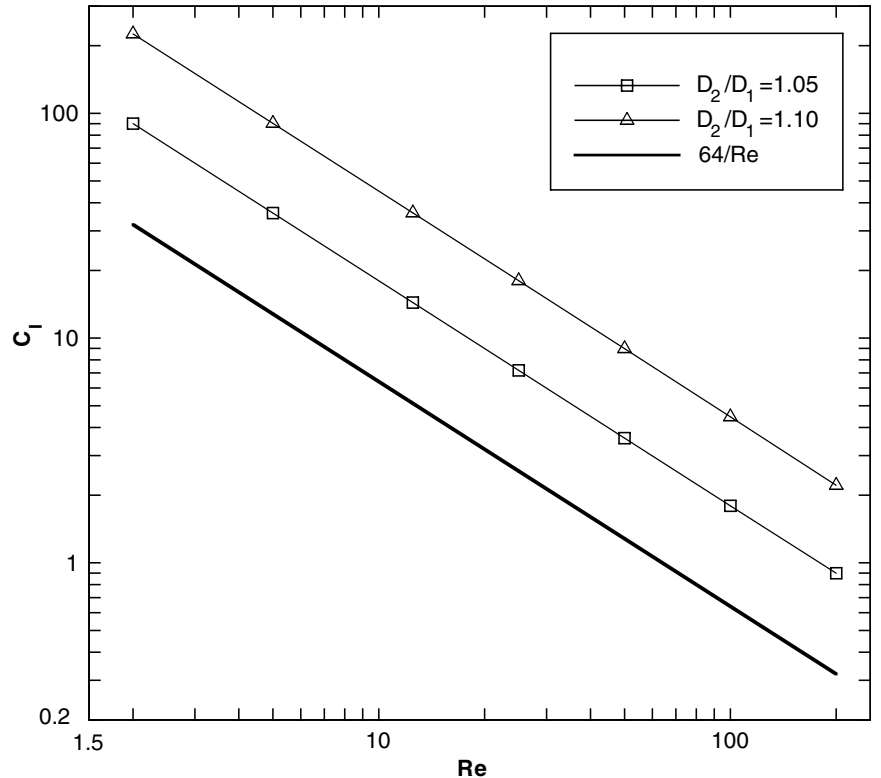

Fig. 3. Evolution of the calculated loss coefficient and comparison with the theory Darcy friction coefficient for laminar flow for a very small expansion angle $\left(\theta=0.5^{\circ}\right)$.

sion $64 / R e$ for fully developed laminar flow in pipes, as the diameter ratio is reduced to 1 for diffusers with $0.5^{\circ}$ halfangle. Calculations for fully developed pipe flow collapsed with the analytical solution.

For the sudden expansion $\left(\theta=90^{\circ}\right)$ our results match those of Oliveira et al. (1998) as is shown in Fig. 4. Here, we also see the progression of $C_{\mathrm{I}}$ as the diffuser angle increases and for $\theta=80^{\circ}$ it is clear that $C_{\mathrm{I}}$ is already very close to that for a sudden expansion. 


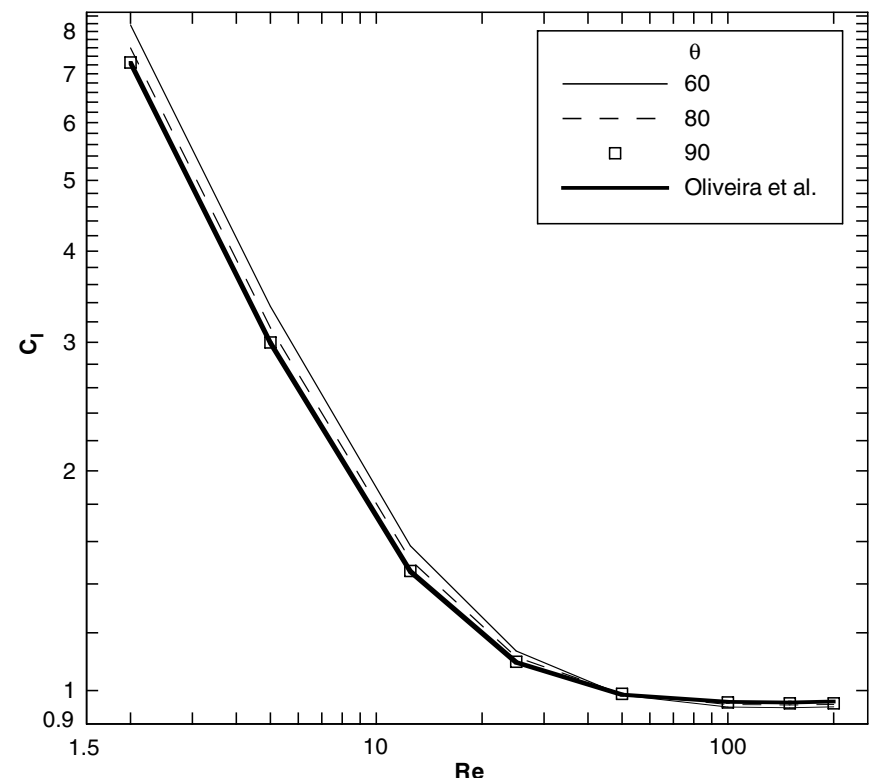

Fig. 4. Evolution of the calculated loss coefficient with Reynolds number for high diffuser half-angles and $D_{2} / D_{1}=2$ and comparison with the correlation of Oliveira et al. (1998) for $\theta=90^{\circ}$.

\section{Results}

The variation of $C_{\mathrm{I}}$ with Reynolds number and diffuser angle for Newtonian fluids is presented in Figs. 5 and 6 for $D_{2} / D_{1}=1.5$ and $D_{2} / D_{1}=2$, respectively. The behaviour of $C_{\mathrm{I}}$ is strongly influenced by diffuser angle and the relation between viscous and inertial forces. As the angle increases $C_{\mathrm{I}}$ decreases at a constant Reynolds number, whereas at a constant diffuser angle $C_{\mathrm{I}}$ decreases with

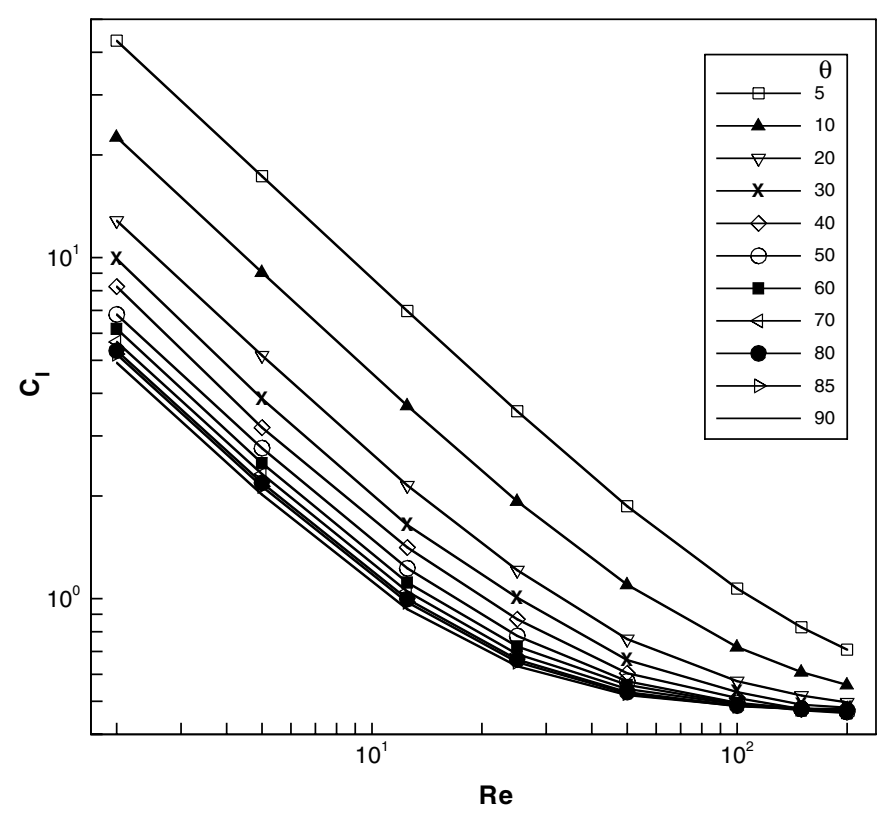

Fig. 5. Calculated $C_{\mathrm{I}}$ as function of Reynolds number and diffuser angle for $D_{2} / D_{1}=1.5$.

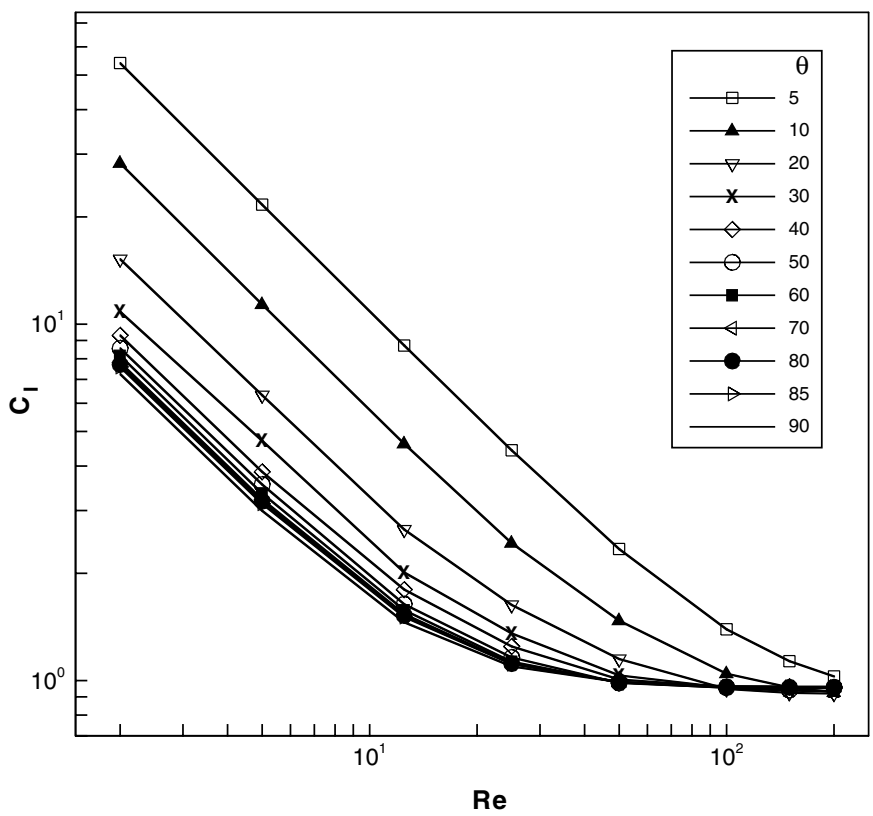

Fig. 6. Calculated $C_{\mathrm{I}}$ as function of Reynolds number and diffuser angle for $D_{2} / D_{1}=2.0$.

increasing Reynolds number. When viscous forces predominate the pressure variation scales with a viscous stress, hence the normalisation with the kinetic energy leads to $C_{\mathrm{I}}$ varying linearly with $1 / R e$. For instance, $C_{\mathrm{I}}$ increases 12 times, from 2.41 to 28.26 when $R e$ decreases from 25 to 2 , for $\theta=10^{\circ}$ and $D_{2} / D_{1}=2$. In contrast, at high-Reynolds numbers the pressure variation scales with the kinetic energy and $C_{\mathrm{I}}$ tends to constant values: for instance, when $R e$ increases from 50 to $200, C_{\mathrm{I}}$ only decreases by a mere $6 \%$, from 1.00 to 0.94 for $\theta=40^{\circ}$ and $D_{2} / D_{1}=2$.

At low-Reynolds numbers, $C_{\mathrm{I}}$ increases significantly when the diffuser angle decreases. As an example, for $D_{2} / D_{1}=2$ and $R e=5$, the value of $C_{\mathrm{I}}$ at $\theta=50^{\circ}$ is $16 \%$ higher than for $\theta=90^{\circ}$, whereas for $\theta=10^{\circ}, C_{\mathrm{I}}$ is $310 \%$ higher than at $\theta=90^{\circ}$. $C_{\mathrm{I}}$ is less sensitive to $\theta$ at large Reynolds numbers, since the separated flow region becomes longer than the diffuser: for $R e=150$ and the same diameter ratio $C_{\mathrm{I}}$ only varies $1 \%$ when $\theta$ increases from $10^{\circ}$ to $90^{\circ}$.

The variations of $C_{\mathrm{I}}$ with $\theta$ may appear in contradiction with common knowledge that diffusers are more efficient the smaller their angle. This stands from $C_{\mathrm{I}}$ accounting for all perturbations to the flow, which take place over different lengths of pipe for different diffuser angles. As $\theta$ increases the diffuser length $L_{2}$ decreases, so the various contributions to $C_{\mathrm{I}}$ vary in opposite directions: for instance, the frictional pressure drop within the diffuser drops to zero at $\theta=90^{\circ}$ but, in contrast, the irreversible loss of energy due to inefficient flow deceleration and the velocity and pressure distortions increase significantly with $\theta$. The relative weight of these contributions depend critically on the Reynolds number and diffuser angle as will be 


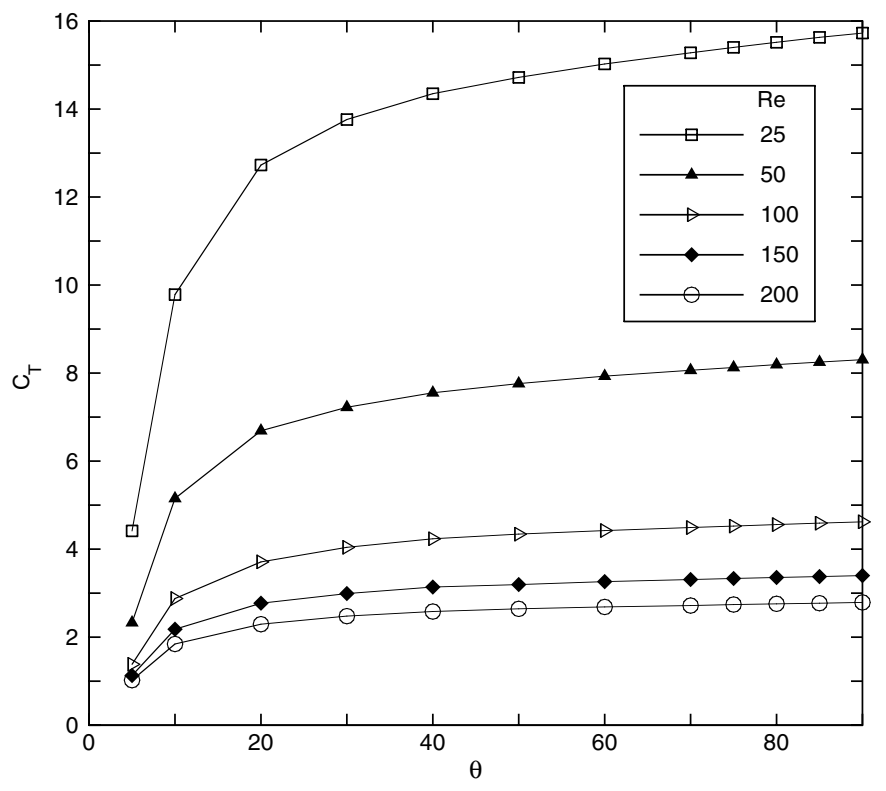

Fig. 7. Variation of total pressure coefficient with diffuser angle and $R e$ for $D_{2} / D_{1}=2$.

shown. To correctly compare the losses in different diffusers, the true pressure variation over the same length of duct must be compared and the flow must be fully-developed at inlet and outlet. This is carried out in Fig. 7, where the normalized total pressure variation $\left(C_{\mathrm{T}}\right)$ is plotted as a function of diffuser angle $\left(\theta \geqslant 5^{\circ}\right)$ for different Reynolds numbers and constant $D_{2} / D_{1}$. Now it is clear that the overall loss is higher for more open diffusers, with a stronger variation of $C_{\mathrm{T}}$ at low angles and Reynolds numbers because of larger variations in flow separation under these conditions. It is possible that for $\theta \leqslant 5^{\circ}$ a minimum in $C_{\mathrm{T}}$ will be observed, because the duct becomes extremely long, thus increasing frictional losses by more than the reduction in the other contributions. We did not perform simulations for $\theta<5^{\circ}$ given the very long computational domains and corresponding large CPU times required.

From the results of the numerical calculations, we can quantify the corrective terms $\Delta C_{\mathrm{F}}, \Delta C_{\beta}, \Delta C_{p 0}$ derived in the approximate 1D-theory and assess their relevance to the corrected coefficient $C_{\mathrm{Ic}}$ given in Eq. (7), which is then compared with the calculated $C_{\mathrm{I}}$ (also called correct or true $\left.C_{\mathrm{I}}\right)$.

The effects of the distorted velocity profiles upstream and downstream of the diffuser are quantified by $\Delta C_{\beta 1}$ and $\Delta C_{\beta 2}$, respectively and depend on the profile shape factors for momentum $\beta_{01}$ and $\beta_{02}$ plotted in Figs. 8 and 9 , respectively. These were determined by numerical integration of the calculated velocity profiles at planes 01 and $02 . \beta_{1}$ decreases with $\theta$ from the fully-developed value of $4 / 3$, with inertia reducing the magnitude of its variation and of the correction $\Delta C_{\beta 1}$ (see Eq. (10)). At the expansion outlet, the behavior is qualitatively different: both $\beta_{02}$ and $\Delta C_{\beta 2}$ increase with $\theta$ and the Reynolds

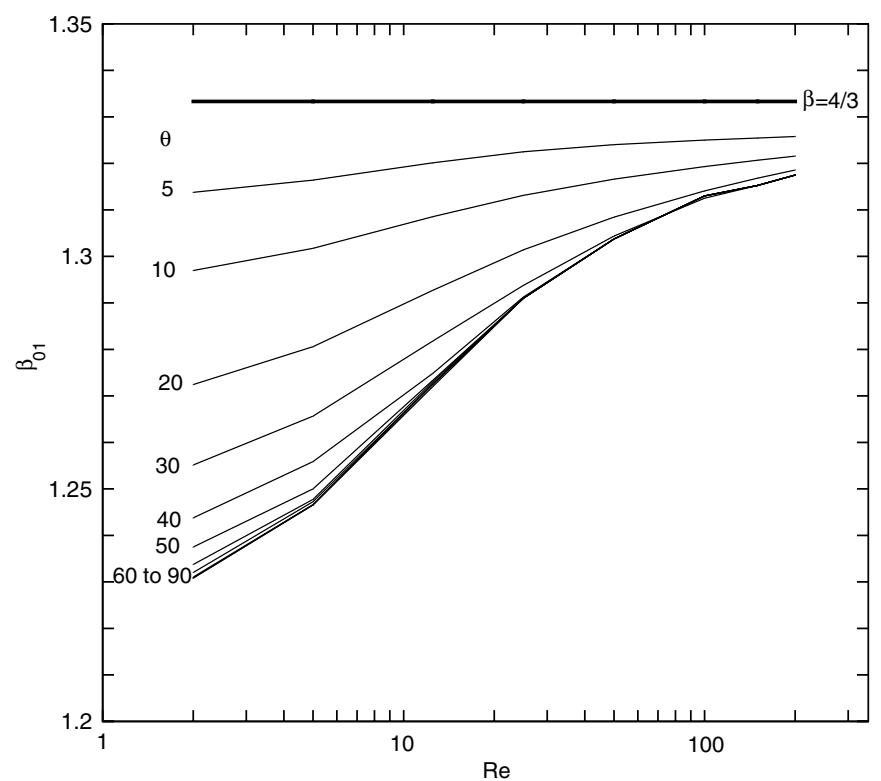

Fig. 8. Variation of momentum shape factor $\beta_{01}$ with Reynolds number and diffuser angle for $D_{2} / D_{1}=2$.

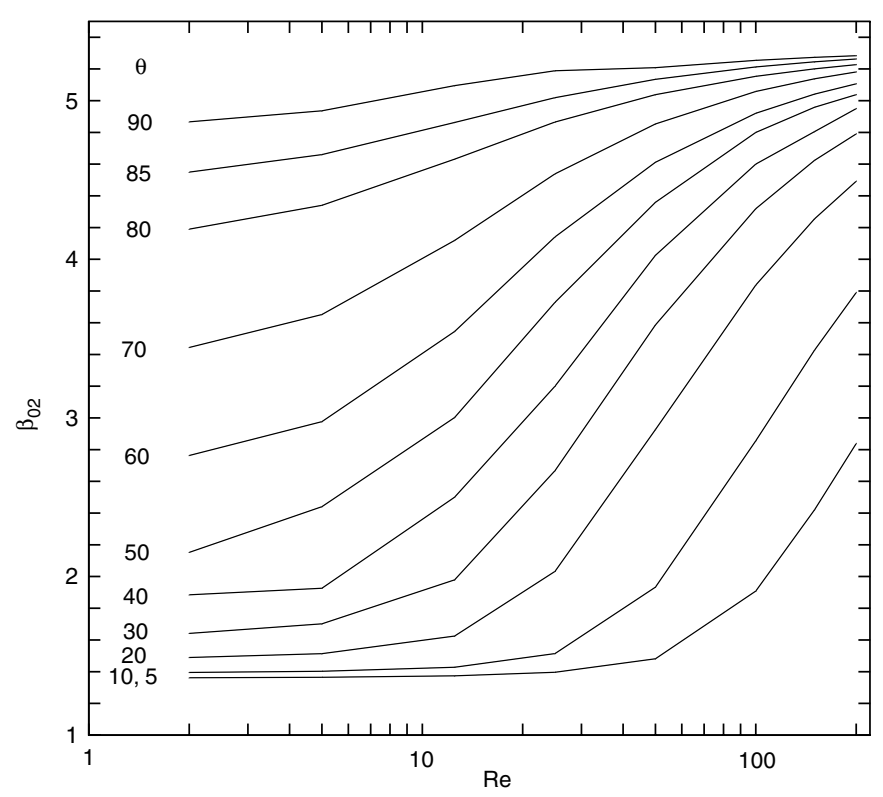

Fig. 9. Variation of momentum shape factor $\beta_{02}$ with Reynolds number and diffuser angle for $D_{2} / D_{1}=2$.

number from the fully-developed values of $\beta=4 / 3$ and 0 , respectively.

Differences between fully developed and actual friction at the inlet $\left(\Delta C_{\mathrm{F} 1}\right)$ and outlet $\left(\Delta C_{\mathrm{F} 2}\right)$ pipes are represented in Figs. 10 and 11, respectively. As inertia dominates the flow $\Delta C_{\mathrm{F} 1}$ decreases to a negligible contribution at high Reynolds numbers, whereas at small Reynolds numbers the distortion of the upstream flow by diffusion leads to values of $\Delta C_{\mathrm{F} 1}$ as important as those of $\Delta C_{\mathrm{F} 2}$, but of opposite sign. At high-Reynolds numbers, the distortion of the 


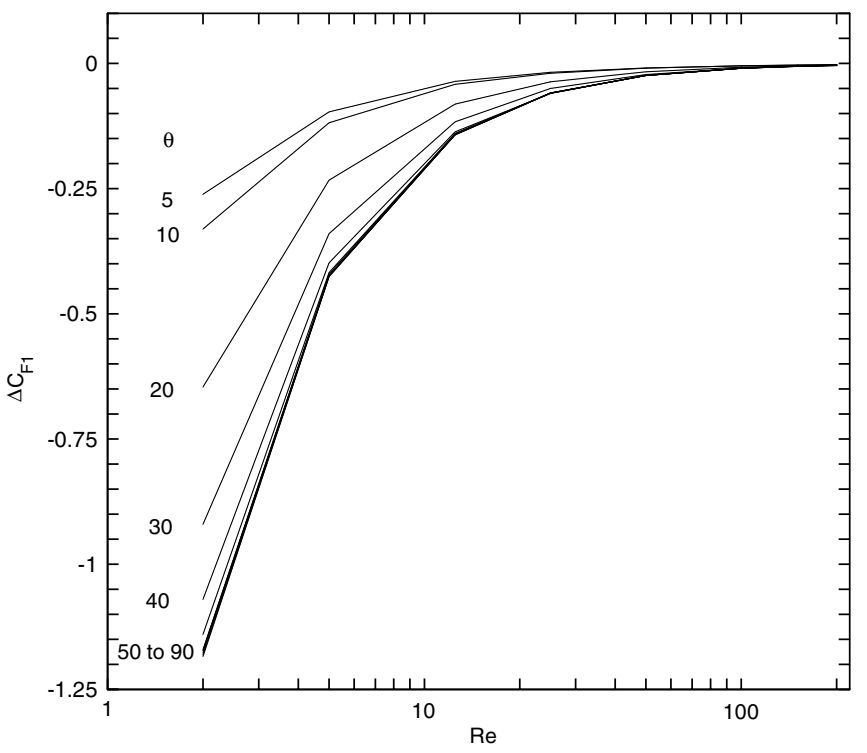

Fig. 10. Variation of $\Delta C_{\mathrm{F} 1}$ with Reynolds number and diffuser angle for $D_{2} / D_{1}=2$.

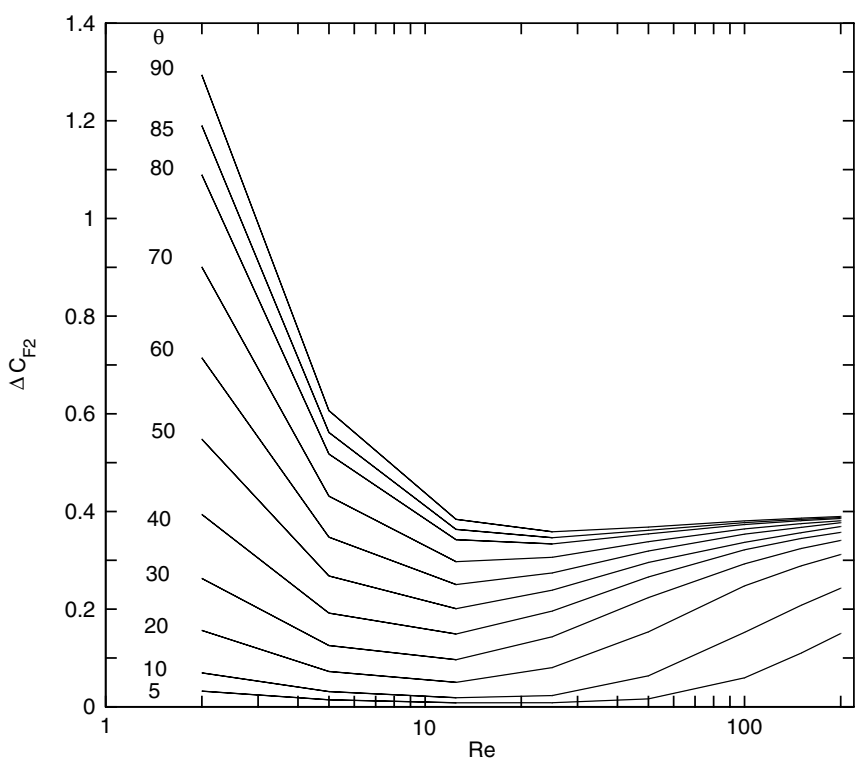

Fig. 11. Variation of $\Delta C_{\mathrm{F} 2}$ with Reynolds number and diffuser angle for $D_{2} / D_{1}=2$.

inlet pipe flow is negligible and the main friction correction comes from the outlet pipe, $\Delta C_{\mathrm{F} 2}$ (Fig. 11), because of the strong recirculation and long development lengths. For diffuser angles larger than $60^{\circ}$ note that there is always a downstream recirculation, even at low Reynolds number flows.

The correction due to non-uniform pressure distributions at the inlet and outlet planes, $\Delta C_{p 0}$, is plotted in Fig. 12 and is especially relevant at low Reynolds numbers because of the role of diffusion in distorting the pressure profiles. At high-Reynolds numbers, in contrast, the flow

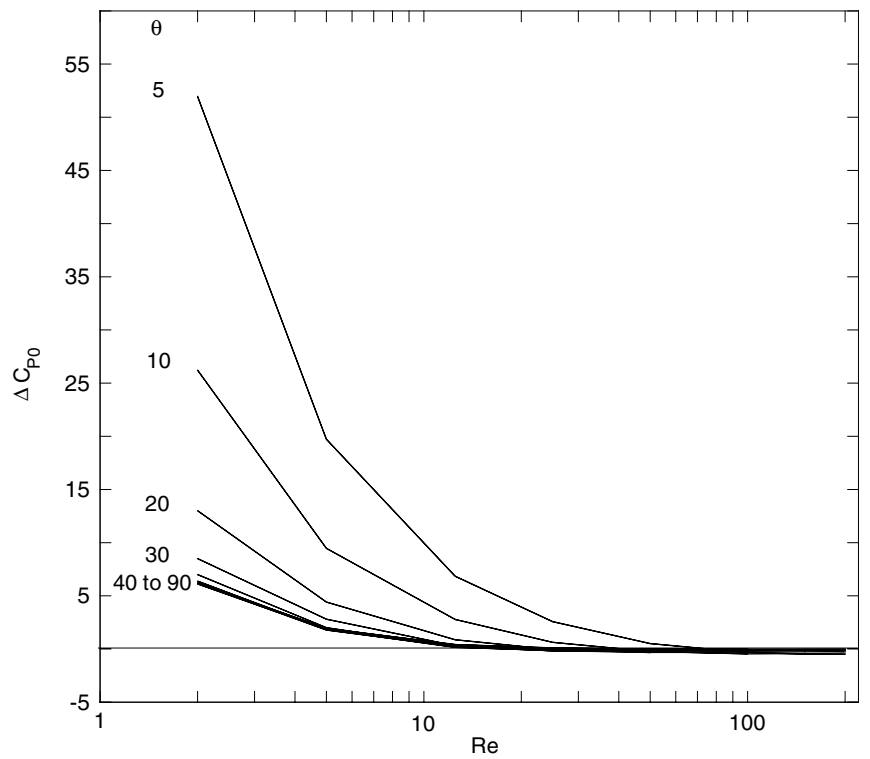

Fig. 12. Variation of $\Delta C_{p 0}$ with Reynolds number and diffuser angle, for $D_{2} / D_{1}=2$.

is parallel to the straight pipes, the pressure variation in the radial direction decreases and the pressure profiles become uniform. As the diffuser angle increases to $45^{\circ}$, $\Delta C_{p 0}$ decreases intensively, but for $\theta>45^{\circ}, \Delta C_{p 0}$ tends to become independent of $\theta$, since the flow downstream the diffuser is basically determined by the recirculation and is insensitive to the expansion wall orientation, as already mentioned.

For some representative diffuser angles and Reynolds numbers, Table 4 compares the values of the true $\left(C_{\mathrm{I}}\right)$ and corrected $\left(C_{\mathrm{Ic}}\right)$ loss coefficients (Eq. (7)), of $C_{\mathrm{R}}$ and of the coefficient usually found in the literature, $C_{\mathrm{Ith}}$ in Eq. (14), including values of the various corrective terms. The differences between the corrected and true loss coefficients are small, never exceeding $1 \%$ for the listed cases, thus confirming the appropriateness of the approximate 1-D theory in spite of its simplifications. Regarding the coefficient of Eq. (14), its value differs significantly from the true $C_{\mathrm{I}}$ (always by more than $50 \%$ ), especially at low Reynolds numbers, and so it can be concluded that it is not an appropriate expression to be used under laminar flow conditions.

Finally, we list in Table 5 all the calculated values of $C_{\mathrm{I}}$ as a function of the Reynolds number and diffuser angle, which constitutes the main deliverable of this work. For ease of use and to compact these data, the correlations of Eq. (17) were derived: for $D_{2} / D_{1}=1.5$ the expression provides values which generally do not differ from those in Table 5 by more than 7\% (it does for the sudden expansion, but here an adequate correlation can be found in Oliveira et al. (1998)), whereas for $D_{2} / D_{1}=2$ the difference between Eq. (17) and data in Table 5 can be as large as $20 \%$. If higher accuracy is necessary we recommend the direct use of Table 5 or fitting the data under more strict conditions (say, for constant diffuser angles). 
Table 4

Predicted $C_{\mathrm{I}}$, corrections and corrected theoretical loss coefficients at the diffuser

\begin{tabular}{|c|c|c|c|c|c|c|c|c|}
\hline$R e$ & $C_{\mathrm{I}}$ & $\left(C_{\mathrm{I}}-C_{\mathrm{Ith}} / C_{\mathrm{Ith}}\right)$ & $\Delta C_{\mathrm{F}}$ & $\Delta C_{\beta}$ & $\Delta C_{p 0}$ & $\Delta C_{\theta}$ & $C_{\mathrm{Ic}}$ & $\left(C_{\mathrm{I}}-C_{\mathrm{Ic}} / C_{\mathrm{Ic}}\right)$ \\
\hline \multicolumn{9}{|c|}{$\theta=10^{\circ}$ and $D_{2} / D_{1}=1.5, C_{I t h}=0.1393, C_{R}=1.605$} \\
\hline 2 & 22.5427 & $16077 \%$ & -0.3779 & 0.0972 & 20.6570 & -1.4656 & 22.5427 & $0.00 \%$ \\
\hline 25 & 1.9254 & $1282 \%$ & 0.0485 & 0.1279 & 0.5000 & -1.4656 & 1.9284 & $0.16 \%$ \\
\hline 150 & 0.6074 & $336 \%$ & 0.2859 & 0.4009 & -0.3091 & -1.4656 & 0.6090 & $0.26 \%$ \\
\hline \multicolumn{9}{|c|}{$\theta=40^{\circ}$ and $D_{2} / D_{1}=1.5, C_{I t h}=0.3086, C_{R}=1.605$} \\
\hline 2 & 8.2848 & $2584 \%$ & -0.5776 & 0.3593 & 6.4000 & -1.2963 & 8.2232 & $0.75 \%$ \\
\hline 25 & 0.8615 & $179 \%$ & 0.2273 & 0.4701 & -0.0375 & -1.2963 & 0.8701 & $0.98 \%$ \\
\hline 150 & 0.4727 & $53 \%$ & 0.4082 & 0.6191 & -0.1030 & -1.2963 & 0.4746 & $0.40 \%$ \\
\hline \multicolumn{9}{|c|}{$\theta=70^{\circ}$ and $D_{2} / D_{1}=1.5, C_{I t h}=0.3086, C_{R}=1.605$} \\
\hline 2 & 5.7004 & $1747 \%$ & 0.6502 & 0.6172 & 5.3254 & -1.2963 & 5.6630 & $0.66 \%$ \\
\hline 25 & 0.6843 & $122 \%$ & 0.37917 & 0.6327 & 0.0945 & -1.2963 & 0.6875 & $0.48 \%$ \\
\hline 150 & 0.4747 & $54 \%$ & 0.44683 & 0.6538 & -0.0266 & -1.2963 & 0.4777 & $0.63 \%$ \\
\hline \multicolumn{9}{|c|}{$\theta=10^{\circ}$ and $D_{2} / D_{1}=2.0, C_{I t h}=0.2540, C_{R}=1.875$} \\
\hline 2 & 28.2570 & $11027 \%$ & -0.2612 & 0.08048 & 26.2260 & -1.6210 & 28.2817 & $0.09 \%$ \\
\hline 25 & 2.4121 & $850 \%$ & 0.0033 & 0.06298 & 0.6214 & -1.6210 & 2.4301 & $0.74 \%$ \\
\hline 150 & 0.9511 & $274 \%$ & 0.2045 & 0.28707 & -0.4266 & -1.6210 & 0.9569 & $0.61 \%$ \\
\hline \multicolumn{9}{|c|}{$\theta=40^{\circ}$ and $D_{2} / D_{1}=2.0, C_{I t h}=0.5625, C_{R}=1.875$} \\
\hline 2 & 9.3119 & $1555 \%$ & -0.6762 & 0.2481 & 7.0000 & -1.3125 & 9.3032 & $0.09 \%$ \\
\hline 25 & 1.2434 & $121 \%$ & 0.1362 & 0.3175 & -0.1750 & -1.3125 & 1.2463 & $0.23 \%$ \\
\hline 150 & 0.9543 & $70 \%$ & 0.3387 & 0.4681 & -0.1075 & -1.3125 & 0.9607 & $0.66 \%$ \\
\hline \multicolumn{9}{|c|}{$\theta=70^{\circ}$ and $D_{2} / D_{1}=2.0, C_{I t h}=0.5625, C_{R}=1.875$} \\
\hline 2 & 7.8206 & $1290 \%$ & -0.2837 & 0.4665 & 6.1145 & -1.3125 & 7.8067 & $0.18 \%$ \\
\hline 25 & 1.1130 & $98 \%$ & 0.2470 & 0.4850 & -0.0244 & -1.3125 & 1.1187 & $0.51 \%$ \\
\hline 150 & 0.9490 & $69 \%$ & 0.3696 & 0.5032 & -0.0475 & -1.3125 & 0.9547 & $0.59 \%$ \\
\hline
\end{tabular}

Table 5

Calculated $C_{\mathrm{I}}$ data

\begin{tabular}{|c|c|c|c|c|c|c|c|c|c|c|c|}
\hline $\operatorname{Re} / \theta$ & 5 & 10 & 20 & 30 & 40 & 50 & 60 & 70 & 80 & 85 & 90 \\
\hline \multicolumn{12}{|c|}{$D_{2} / D_{1}=1.5$} \\
\hline 2 & 43.2391 & 22.5427 & 12.8351 & 9.9301 & 8.2848 & 6.8179 & 6.1918 & 5.7004 & 5.3449 & 5.2200 & 4.9270 \\
\hline 5 & 17.3225 & 9.0438 & 5.1668 & 3.8648 & 3.1839 & 2.7589 & 2.4998 & 2.3310 & 2.1947 & 2.1307 & 2.0222 \\
\hline 12.5 & 6.9670 & 3.6746 & 2.1443 & 1.6338 & 1.4116 & 1.2224 & 1.1091 & 1.0530 & 0.9969 & 0.9728 & 0.9350 \\
\hline 25 & 3.5421 & 1.9254 & 1.2068 & 0.9992 & 0.8615 & 0.7725 & 0.7221 & 0.6843 & 0.6597 & 0.6491 & 0.6301 \\
\hline 50 & 1.8620 & 1.0934 & 0.7561 & 0.6581 & 0.5972 & 0.5686 & 0.5557 & 0.5405 & 0.5304 & 0.5257 & 0.5183 \\
\hline 100 & 1.0656 & 0.7194 & 0.5687 & 0.5312 & 0.5063 & 0.4913 & 0.4884 & 0.4865 & 0.4849 & 0.4831 & 0.4841 \\
\hline 150 & 0.8223 & 0.6074 & 0.5175 & 0.4882 & 0.4727 & 0.4708 & 0.4686 & 0.4747 & 0.4723 & 0.4737 & 0.4745 \\
\hline 200 & 0.7079 & 0.5572 & 0.4939 & 0.4804 & 0.4725 & 0.4620 & 0.4609 & 0.4646 & 0.4674 & 0.4675 & 0.4738 \\
\hline \multicolumn{12}{|c|}{$D_{2} / D_{\mathrm{I}}=2.0$} \\
\hline 2 & 53.9367 & 28.2570 & 15.1903 & 10.7859 & 9.3119 & 8.5715 & 8.1662 & 7.8206 & 7.5951 & 7.4853 & 7.2540 \\
\hline 5 & 21.6133 & 11.3354 & 6.3392 & 4.7151 & 3.8608 & 3.5533 & 3.3605 & 3.2298 & 3.1416 & 3.0954 & 2.9988 \\
\hline 12.5 & 8.6936 & 4.6084 & 2.6179 & 2.0021 & 1.7960 & 1.6408 & 1.5788 & 1.5386 & 1.5096 & 1.4967 & 1.4584 \\
\hline 25 & 4.4149 & 2.4121 & 1.6137 & 1.3477 & 1.2434 & 1.1624 & 1.1331 & 1.1130 & 1.1116 & 1.1103 & 1.0951 \\
\hline 50 & 2.3298 & 1.4654 & 1.1337 & 1.0166 & 1.0004 & 0.9835 & 0.9853 & 0.9822 & 0.9896 & 0.9914 & 0.9897 \\
\hline 100 & 1.3831 & 1.0352 & 0.9323 & 0.9385 & 0.9595 & 0.9519 & 0.9494 & 0.9490 & 0.9568 & 0.9608 & 0.9632 \\
\hline 150 & 1.1280 & 0.9511 & 0.9196 & 0.9218 & 0.9543 & 0.9360 & 0.9471 & 0.9490 & 0.9554 & 0.9565 & 0.9605 \\
\hline 200 & 1.0221 & 0.9234 & 0.9055 & 0.9262 & 0.9438 & 0.9488 & 0.9499 & 0.9475 & 0.9569 & 0.9567 & 0.9604 \\
\hline
\end{tabular}

$$
\left\{\begin{aligned}
C_{\mathrm{I}}= & \frac{11.1(\sin \theta)^{-0.824}}{R e^{-2.23(\sin \theta)^{3}+2.98(\sin \theta)^{2}-0.874(\sin \theta)+1.04}+81.6(\sin \theta)^{4}-213(\sin \theta)^{3}+180(\sin \theta)^{2}-52.6(\sin \theta)} & \\
& +3.01\left(-75.8(\sin \theta)^{4}+196(\sin \theta)^{3}-166(\sin \theta)^{2}+50(\sin \theta)-3.13\right) \log R e & \\
& +\left(17.7(\sin \theta)^{4}-45.4(\sin \theta)^{3}+38.5(\sin \theta)^{2}-11.86(\sin \theta)+0.851\right)(\log R e)^{2}, & D_{2} / D_{1}=1.5 \\
C_{\mathrm{I}}= & \frac{16.4(\sin \theta)^{-0.703}}{R^{1.54(\sin \theta)^{3}+3.33(\sin \theta)^{2}-2.24(\sin \theta)+1.24}+202(\sin \theta)^{4}-559(\sin \theta)^{3}+550(\sin \theta)^{2}-217(\sin \theta)} & \\
& +21.9\left(-173(\sin \theta)^{4}+470(\sin \theta)^{3}-456(\sin \theta)^{2}+179(\sin \theta)-18.3\right) \log R e & \\
& +\left(37.1(\sin \theta)^{4}-99.6(\sin \theta)^{3}+96(\sin \theta)^{2}-37.8(\sin \theta)+4.06\right)(\log R e)^{2}, & D_{2} / D_{1}=2
\end{aligned}\right.
$$




\section{Conclusions}

An extensive set of numerical calculations was carried out for laminar Newtonian fluid flow in diffusers at Reynolds numbers from 2 to 200 , diffuser angles from $5^{\circ}$ to $90^{\circ}$, and two different diameter ratios of 1.5 and 2 in order to quantify the loss coefficient, which is listed in Table 5 with an estimated uncertainty of less than $3 \%$. A correlation fitting these data with differences never exceeding $7 \%$ is also presented in Eq. (17) for ease of use. The $C_{\mathrm{I}}$ data show a strong dependence on the Reynolds number with $C_{\mathrm{I}}$ increasing as $R e$ and diffuser angle both decrease, but increasing with radius ratio.

The simplified 1-D theory of Oliveira and Pinho (1997) for sudden expansions was generalized to a diffuser and used to help understand the observed variations of $C_{\mathrm{I}}$ in terms of corrections to the reversible loss coefficient. The differences between $C_{\mathrm{I}}$ and $C_{\mathrm{R}}$ or $C_{\mathrm{I}}$ th are accounted for by the role of diffusion in distorting velocity and pressure profiles and by differences between real and fully-developed friction. Large discrepancies were found between the reversible and the calculated loss coefficients and between the calculated loss coefficient and the values given by an often-quoted expression from the literature, which is not adequate for laminar flows given its assumptions of inviscid flow with uniform fully-developed profiles at inlet and outlet.

\section{Acknowledgements}

Both authors wish to thank Prof. P. J. Oliveira of Universidade da Beira Interior (Portugal) for making available the original computer code. S. Rosa also wishes to thank the sponsorship of company Navegadores-Ensino de Informática, Lda, for funding part of this work.

\section{References}

Crane Co., 1979. Flow of fluids. Technical Paper 410, Chicago.

Gibson, A.H., 1930. Hydraulics and Its Applications, fourth ed. Van Nostrand Co., Berrlin.

Issa, R.I., Oliveira, P.J., 1992. Numerical predictions of phase separation in two-phase flow through $T$-junctions. Comput. Fluids 23, 347-372.

Khosla, P.K., Rubin, S.G., 1974. A diagonally dominant second-order accurate implicit scheme. Comput. Fluids 2, 207-209.

Massey, B.S., 1989. Mechanics of Fluids, sixth ed. Chapman \& Hall, London, p. 213.

Oliveira, P.J., 1992. Compute modelling of multidimensional multiphase flow and application to $T$-junctions. Ph.D. thesis, Imperial College, London, UK.

Oliveira, P.J., Pinho, F.T., 1997. Pressure drop coefficient of laminar Newtonian flow in axisymmetric sudden expansions. Int. J. Heat Fluid Flow 18, 518-529.

Oliveira, P.J., Pinho, F.T., Schulte, A., 1998. A general correlation for local loss coefficient in Newtonian axisymmetric sudden expansions. Int. J. Heat Fluid Flow 19, 655-660.

Patankar, S.V., 1980. Numerical Heat Transfer and Fluid Flow. Hemisphere Publishing Company, Washington.

Peric, M., 1985. A finite volume method for the prediction of threedimensional fluid flow in complex duct. Ph.D. thesis, Imperial College, University of London.

Richardson, L.F., 1927. The deferred approach to the limit. Trans. R. Soc. London Ser. A 226, 229-361.

Runstadler, P.W. et al., 1975. Diffuser Data Book, Creare Inc. Tech. Note 186, Hanover.

Shames, I.H., 1992. Mechanics of Fluids, third ed. McGraw-Hill International Editions, Berlin, p. 370.

Tsui, Y.-Y., Wang, C.-K., 1995. Calculation of laminar separated flow in symmetric two-dimensional diffusers. Trans. ASME 117, 612-616.

Van Doormal, J.P., Raithby, G.D., 1984. Enhancements of the SIMPLE method for predicting incompressible fluid flows. Numer. Heat Transfer 7, 147-163. 